on-screen display of measurements during endoscopy will make it easier, and encourage more endoscopists to record with more precision the location and size of any landmarks or lesions found.

\section{PTU-55 BILIARY STONE CHARACTERISTICS DETERMINING DIFFICULTY AT CONVENTIONAL ERCP AND NEED FOR CHOLANGIOSCOPY-ASSISTED LITHOTRIPSY}

${ }^{1}$ Akhilesh Mulay*, ${ }^{1}$ Tareq El-Menabawey, ${ }^{1}$ Simon Philpotts, ${ }^{1}$ David Graham, ${ }^{1}$ Harry Martin ${ }^{2}$ Amrita Sethi, 'George Webster. 'University College London Hospital, London, UK; ${ }^{2}$ Columbia University Medical Centre, New York, USA

\subsection{6/gutjnl-2021-BSG.128}

Introduction Stone clearance at ERCP is suboptimal, with NHS data suggesting 52\% of ERCPs for stones are repeat procedures. ${ }^{1}$ Cholangioscopy-assisted lithotripsy is recommended for treating difficult bile duct stones (ESGE guidelines), but the characteristics of difficult stones are poorly defined, and patients may be undergoing conventional ERCP with limited chance of stone clearance. We sought to determine these characteristics and explore decision making in biliary endoscopy.

Methods 53 biliary endoscopists independently assessed 20 cholangiograms with biliary stones (16 ERCPs, 4 MRCPs). Using Likert-type questionnaires each image was graded in 3 areas; 'grading of stone difficulty', 'confidence of clearance with conventional ERCP methods' and 'likelihood of needing cholangioscopy-assisted lithotripsy'. Images were objectively assessed according to stone characteristics: 'largest stone size', 'stone number', 'presence of stricture distal to stone', 'size of stone relative to distal duct size'. Univariate analyses were performed to determine the effect of these characteristics on endoscopists' questionnaire responses.

Results Largest stone size significantly affected endoscopists' grading of difficulty, confidence of clearance with conventional ERCP methods and likelihood of needing cholangioscopy $(\mathrm{p}=$ $<0.01$ for all, Kruskal-Wallis (KW) test). As stone size increased, particularly $>15 \mathrm{~mm}$, endoscopists rated them as more difficult, were less confident with conventional methods and were more likely to need cholangioscopy. Presence of stricture distal to stone significantly affected responses to all 3 areas ( $\mathrm{p}=<0.01$ for all, $\chi^{2}$ test), as did size of stone relative to distal duct $(\mathrm{p}=<0.01$ for all, KW test). The presence of a stricture distal to a stone, and a larger stone relative to distal duct showed a clear trend in more difficult ratings, less confidence with conventional methods and a greater likelihood of requiring cholangioscopy. Stone number significantly affected responses to all 3 areas $(\mathrm{p}=<0.01$ for all, KW test), however the presence of only one stone led to more difficult ratings, less confidence with conventional methods and an increased likelihood of using cholangioscopy.

Conclusions Larger stone size, the presence of a stricture distal to a stone, a larger stone relative to the size of the distal duct and fewer stones result in endoscopists grading stones as more difficult and being more likely to use cholangioscopy. The result for stone number is unexpected and warrants further analysis. Our results suggest that pre-procedure stone characteristics may allow stratification of patients for advanced ERCP techniques.

\section{REFERENCE}

1. Martin, $\mathrm{H}$, et al/The national performance in the management of common bile duct stones in England. Gut 2021;70:A66.

\section{PTU-56 UPPER GASTROINTESTINAL BLEED (UGIB); HEMOSPRAY, AN ESSENTIAL TOOL IN THE ARMAMENTARIUM}

Siva Selvan*, Vignesh Balasubaramaniam, Dhanoop Mohandas, Shailesh Karanth. Queen Elizabeth Hospital King's Lynn, King's Lynn, UK

\subsection{6/gutjnl-2021-BSG.129}

Introduction Upper gastrointestinal bleed (UGIB) is a common presentation to the emergency department and accounts for approximately 50,000 - 70,000 admissions per year in the UK.Peptic ulcer disease (PUD) remains the most common cause of UGIB in the UK. Hemospray (Cook Medical, Winston-Salem, NC, USA) is an inert powder developed for endoscopic haemostasis. We aim to appraise the outcomes for UGIB where hemospray was used during the initial endoscopic therapeutic intervention.

Methods In this retrospective study from March 2018 to December 2020, cases of severe UGIB intervened with hemospray during primary presentation were identified via HICCS, an online database of endoscopy procedures. A detailed analysis of the demographics and outcome measures relating to the procedure, anatomical site of intervention, re-bleeding, and 30-day mortality were collected and interpreted.

Results 20 patients with severe UGIB were identified, where hemospray was used to control the bleeding when other modalities such as Adrenaline injection, Endoclip and Gold probe application failed to stop the bleeding. Among this population, the mean age was 73 years, ranging between 61 to 98 years. There were 14 male patients $(70 \%)$ in this cohort. Majority of the therapeutic intervention site was at the duodenum ( $\mathrm{n}=14)$ accounting for $70 \%$ of the patients, followed by stomach in $25 \%(\mathrm{n}=5)$ and oesophagus $(\mathrm{n}=1)$ in $5 \%$. Causes for the severe UGIB were duodenal ulcer in $12(60 \%)$, gastric ulcer in $2(10 \%)$, gastric malignancy in $2(10 \%)$, Dieulafoy lesion in $1(5 \%)$, metastatic duodenal tumour in $1(5 \%)$, Angiodysplasia in $1(5 \%)$ and severe reflux oesophagitis in $1(5 \%)$. Hemospray was successful in achieving initial haemostasis in all cases except in one case of massive haemorrhage with poor view of the bleeding site in duodenum where the bleeding could not be stopped. Following initial hemostasis, re-bleed occurred in 4 out of 19 patients (21\%) who were then managed with best supportive care as treatment escalation was not considered appropriate due to comorbidity. 7 patients (35\%) died within 30 days of the procedure out of which four occurred due to re-bleed and three patients died due to other medical causes.

Conclusions In our experience, Hemospray has proven to be an effective therapeutic intervention in achieving haemostasis in cases of severe UGIB when other endoscopic therapies fail to stop bleeding.

\section{REFERENCE}

1. Dinesen L, Benson, M., 2012. Managing acute upper gastrointestinal bleeding in the acute assessment unit. Clinical medicine, 12(6), p.589. https://www.hicssuhs. co.uk/about/ 